\title{
Transfusion Risk for Hepatitis B, Hepatitis C and HIV in the State of Santa Catarina, Brazil, 1991-2001
}

Emil Kupek

\author{
Department of Public Health, Federal University of Santa \\ Catarina, Florianópolis, SC, Brazil
}

We examined the time trend of residual risk of transfusing blood contaminated with HIV, hepatitis B and hepatitis C, in the largest blood bank of the state of Santa Catarina, Brazil, 19912001. The HIV risk was reduced approximately 10 times during the decade of 1990, to 1:48777, but then increased significantly by the end of 2001. A similar time trend was observed for hepatitis $B$ and hepatitis $C$, although the increase was not significant in this case. Even during the period of lowest risk, the estimates were considerably higher than in developed countries.

Key Words: Blood transfusion, HIV, hepatitis B, hepatitis C.

Despite the fact that hepatitis B and C are the most frequent infections associated with blood transfusion [1], it was the HIV epidemic that alerted the general public to the importance of serologic tests in blood banks. Few Brazilian studies have looked at the risk of not detecting blood contaminated by these viruses due to the infectious window period, usually denominated as residual (transfusion) risk; they found high risk levels in the states of São Paulo [2] and Santa Catarina [3,4]. We found a surprising upturn of residual risk after a decade of reduction, in the biggest blood bank in the state of Santa Catarina, based on a comparison with previously published results $[3,4]$.

\section{Materials and Methods}

An incidence/window model, applied in large impact studies of this kind in the United Sates [1] and replicated worldwide, including in Brazil [2-4], was used to Received on 20 November 2003; revised 13 April 2004. Address for correspondence: Dr. Emil Kupek. Departamento de Saúde Pública, CCS. Universidade Federal de Santa Catarina. Campus Universitário, Trindade. Zip code: 88040-900 Florianópolis-SC, Brazil. Phone: (048) 334-8654. Fax: (048) 3319542.E-mail: kupek@repensul.ufsc.br

The Brazilian Journal of Infectious Diseases 2004;8(3):236-240 (C) 2004 by The Brazilian Journal of Infectious Diseases and Contexto Publishing. All rights reserved. estimate the residual risk for HIV, hepatitis B and hepatitis $\mathrm{C}$. An estimate is made of the probability of seroconversion within an infectious window period when routine screening tests negative. It is a product of disease incidence and duration of the window period. In a blood bank setting, the incidence can be calculated for repeat donors judged apt on previous donation.

Blood donor records were analyzed for the 19912001 period. During this decade, 22,510 repeat donors with 16,680 person-years of follow-up between the last two donations were the subset for this analysis. The period was divided into four separate phases due to the introduction of new serologic tests with a shorter window period and more rigorous selection of blood donors introduced during the 1990 decade. As a result, all calculations were performed separately for the beginning of the decade (1991-1994), mid decade (1995 to 1996) and the end of decade (1997 to June $17,1999)$, as well as for an update period from June 18, 1999 to 31 December 2001. The date of June 17, 1999 marks the end of the first data collection period rather than any substantial change in serologic tests or donor selection.

In order to minimize the effect of dropout for the confirmatory serologic tests offered to the donor candidates who tested positive in any of the screening tests, the proportion of confirmed cases was extrapolated to the dropout fraction. Although this 
procedure cannot compensate for possible selective dropout, it improves incidence estimates if the latter were similar to the donors who underwent both routine screening and confirmatory tests.

As hepatitis B incidence based on the surface antigen (HbsAg) is only biased downwards, an adjustment for the probability of primary antibody reaction was applied [1]. More details on the methods used can be found in the literature $[1,3,4]$.

The Poisson distribution was used to calculate the $95 \%$ confidence intervals for residual risk estimates.

\section{Results}

The main results are presented in Figure 1.

\section{Discussion}

During the 1990s, there was a considerable reduction of residual risk for HIV, hepatitis $\mathrm{B}$ and hepatitis $\mathrm{C}$ (Figure 1). The HIV risk was reduced approximately 10 times, to 0.0000205 , or $1: 48777$ donors, by the middle of 1999 , but it increased significantly by the end of 2001. A similar decreasing trend for hepatitis B and hepatitis C continued for most of the decade, followed by an upturn in the subsequent period. However, the upturn was not significant for either type of hepatitis. Even during the period of the lowest residual risk, this was still considerably higher than in the developed world, with probability estimates of 0.00048 , or 1:2077 blood donors for hepatitis B, and 0.000073 , or 1:13721 blood donors for hepatitis $\mathrm{C}$. The same was true for the HIV residual risk, which was almost 10 times higher than in developed countries $[1,5]$.

A large decrease in HBV, HIV, and particularly $\mathrm{HCV}$, incidence among repeat blood donors in late 1990s was the most important factor in the risk reduction observed $[3,4]$. During this period, more volunteer donors, more women and more young people were recruited for blood donation. At the same time, additional efforts were made during the predonation interview to improve the detection of behavioral risks for HIV, which largely coincides with those for hepatitis $\mathrm{B}$ and $\mathrm{C}$. Another important factor was a reduction of the window period for HBC antibody from 82 to 52 days in 1996, when this third generation enzyme immunoassay was introduced in this blood bank. Despite this improvement, the residual risk for hepatitis C was still seven times the estimated risk in the United States in the early 1990s [1].

In the absence of reliable prevalence estimates for HIV, hepatitis B and hepatitis C among blood donor candidates in the area $[3,4]$, better selection of blood donors and reduction of the window period upon introduction of a new generation of screening tests may explain the residual risk reduction observed during the 1990s. However, the upturn which followed was a surprise finding. Some health professionals of the blood bank hypothesized that a small group of repeat blood donors could have used the screening tests to verify their HIV status, as this was the fastest way of obtaining the test result, and with no cost to the person tested. This would be the most dangerous scenario, because it greatly increases the risk of a false negative test result due to a greater chance of doing the test during the window period. The latter follows from a supposition that a person who engages in this behavior has been exposed to the risk of HIV only recently and is anxious to verify whether contamination took place on that occasion.

Despite important advances in serological screening of blood donor candidates, some limitations of the donor selection process in identifying high-risk candidates still remain. For example, the large percentage of dropouts for the confirmation test, particularly in the early 1990s, when it reached a level of $78.6 \%$, provokes suspicion about the true motives of blood donation. HIV testing through blood donation could be discouraged by shortening the time in which the Anonymous Testing Center (ATC), established in Florianopolis in 1996 to offer anonymous and costfree HIV testing and counseling, releases the test results. The time was three weeks in ATC and two weeks in the blood bank, thus making the latter a faster track for large sections of a population without means to pay for the test. 
Figure 1. Probability of HIV, hepatitis C and hepatitis B contamination not detected by screening and associated $95 \%$ confidence intervals by period
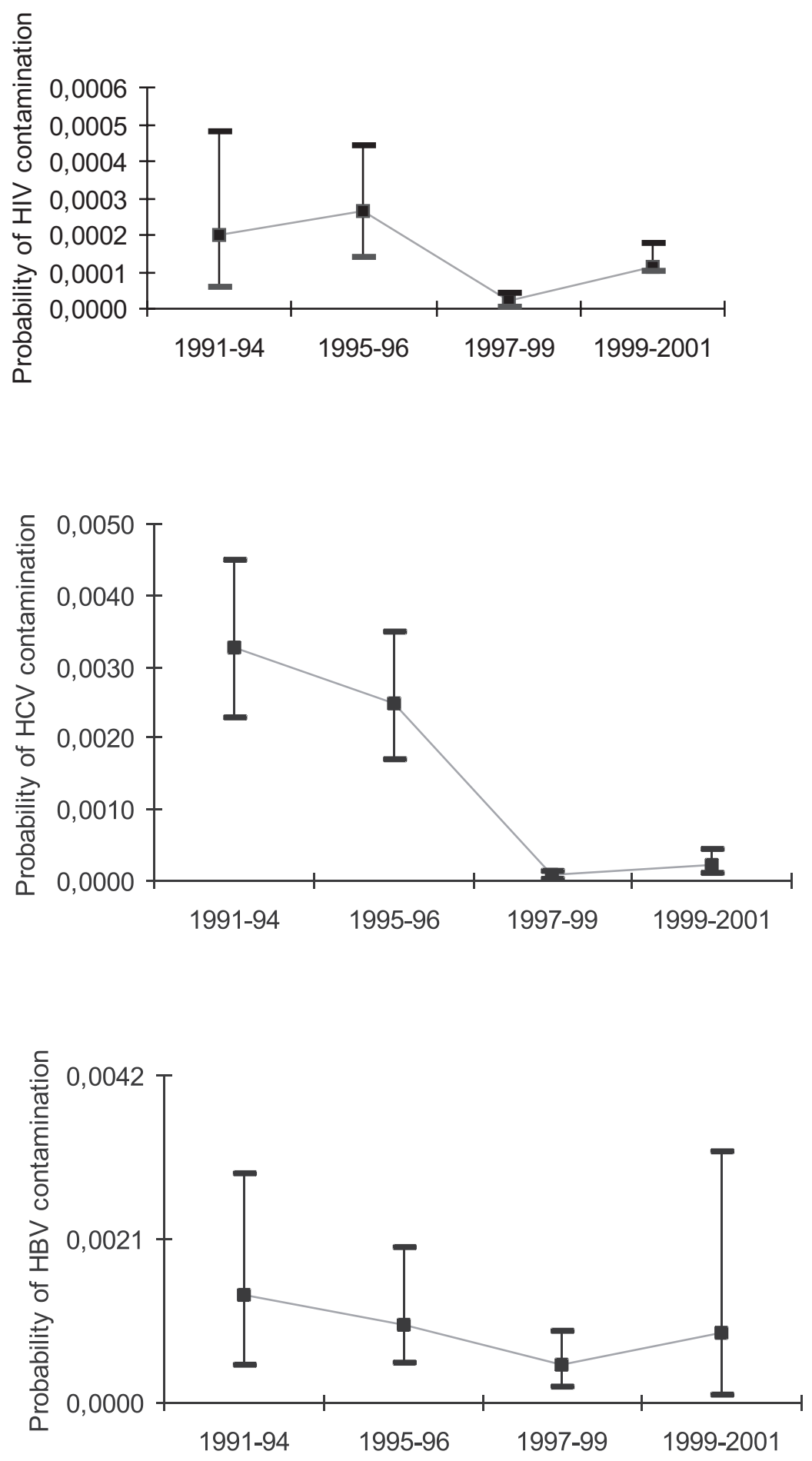

www.bjid.com.br 
The state of Santa Catarina leads the national ranking of the percentage of injectable drug users (IDU) among AIDS patients, with almost a third of all cases being infected in this way, compared to the national average of less than one fifth, during the 1980 to 2002 period [6]. The participation of IDU in total AIDS cases in Santa Catarina compared to the national average decreased from about two times as many in the beginning of the $1990 \mathrm{~s}$, to approximately $50 \%$ more at the beginning of the following decade. Although IDUs are unlikely blood donors, their impact on the spread of HIV in the state as a whole and particularly in its capital, where the main blood bank is situated, has been significant. If their test seeking behavior targeted the blood bank, even a few IDU could have considerably increased the residual risk due to an extremely high incidence of HBV, HCV and HIV in this population.

More educational resources should be made available to explain to the donor candidates the contamination risk for the recipients of blood products due to the infectious window period. Another policy implication is to consider using an RNA polymerase chain reaction (PCR) test for pooled blood screening in high-risk areas, as it can reduce window period to 11 days [1], providing $50 \%$ residual risk reduction compared to the current screening tests. The cost-effectiveness of PCR may be enhanced by also including screening for hepatitis B and hepatitis C [4/5], both of which are highly prevalent among blood donors [3,4]. Recent guidelines of the Brazilian Ministry of Health adopted this strategy to be implemented in the year 2004, although it seems likely that financial and logistic difficulties will delay it for many blood banks. On the other hand, vaccination against hepatitis B and modification of blood donors' risk behavior remain priorities from the public health point of view. Vaccination is now obligatory for all people under 15 years of age and for some high-risk groups, but considerably more efforts are needed to implement it fully and to bring about a significant reduction of hepatitis B prevalence in the country. Preadolescence seems to be the best age to start education about blood-borne infectious diseases, transfusion risks and risky sexual behavior.

Previously established national screening programs for HIV and syphilis in pregnant women could be extended to hepatitis $\mathrm{B}$ and $\mathrm{C}$ at relatively low cost, with large benefits in preventing vertical transmission, as well as sexual transmission of women and their partners. Counseling and information on treatment and prevention should also be provided within the program.

Perhaps the most important lesson to learn from this data is that no amount of sophistication in screening technology can substitute for immunization and education efforts. Although this blood bank acquired better screening tests and improved donor selection by recruiting more volunteer donors and more women, the residual transfusion risk remains vulnerable to HIV test seeking behavior of a small group of donors who use blood donation to check their HIV status. It seems perverse that gains in quality and efficiency of blood screening can be misused in this way by attracting some high risk donors who may not be aware of the risks their behavior represents to the blood recipients. Monitoring residual transfusion risk is a low cost measure for identifying problems of this sort and for taking necessary action to minimize their negative impact on the safety of blood products.

\section{Acknowledgements}

The author wishes to thank all people from the HEMOSC blood bank who helped collecting the data and provided information on donor selection process. This research received financial support from the Brazilian Ministry of Science and Technology $(\mathrm{CNPq}$ grant number 300741/97-9).

\section{References}

1. Schreiber G.B., Busch M.P., Kleinman S.H. The risk of transfusion-transmitted viral infections. New Eng J Med 1996; 334:1685-90. 
2. Canutti Júnior V. Risco transfusional: Metodologia e estudo. In: Covas D.T., Zago M.A. eds. Atualização em hemoterapia. Série de Monografias da Escola Brasileira de Hematologia. Ribeirão Preto-SP: Fundação Hemocentro de Ribeirão Preto, 1998.

3. Kupek E. The reduction of HIV transfusion risk in southern Brazil in the 1990s. Transf Med 2001; 11(2):75-8.

4. Kupek E. Residual transfusion risk for hepatitis B and C in southern Brazil, 1991-99. J Viral Hepat 2001; 8(1):78-82.

5. Roth W.K., Weber M., Seifried E. Feasibility and efficacy of routine PCR screening of blood donations for hepatitis $\mathrm{C}$ virus, hepatitis $\mathrm{B}$ virus, and HIV-1 in a bloodbank setting. Lancet 1999; 353:359-3.

6. Ministério de Saúde - Programa Nacional de DST e AIDS. Tabela IV - Casos de aids, segundo ano de diagnóstico e categoria de exposição hierarquizada. Brasil, 1980- a 2002. Boletim epidemiológico 2003; abril a dezembro de 2002. URL: http:// www.aids.gov.br [accessed 7 September 2003]. 